PROCEEDINGS OF THE

AMERICAN MATHEMATICAL SOCIETY

Volume 125, Number 1, January 1997, Pages 111-119

S 0002-9939(97)03504-1

\title{
AN ABSTRACT ERGODIC THEOREM AND SOME INEQUALITIES FOR OPERATORS ON BANACH SPACES
}

\author{
YUAN-CHUAN LI AND SEN-YEN SHAW
}

(Communicated by Palle E. T. Jorgensen)

\begin{abstract}
We prove an abstract mean ergodic theorem and use it to show that if $\left\{A_{n}\right\}$ is a sequence of commuting $m$-dissipative (or normal) operators on a Banach space $X$, then the intersection of their null spaces is orthogonal to the linear span of their ranges. It is also proved that the inequality $\|x+A y\| \geq$ $\|x\|-2 \sqrt{\|A x\|\|y\|}(x, y \in D(A))$ holds for any $m$-dissipative operator $A$. These results either generalize or improve the corresponding results of Shaw, Mattila, and Crabb and Sinclair, respectively.
\end{abstract}

\section{INTRODUCTION}

Let $X$ be a real (or complex) Banach space and $B(X)$ the Banach algebra of all bounded linear operators on $X$. Given a family $\mathbf{A}$ of closed linear operators on $X$, a net $\left\{A_{\alpha}\right\}$ in $B(X)$ will be called an $\mathbf{A}$-ergodic net if the following conditions hold:

(a) There is an $M>0$ such that $\left\|A_{\alpha}\right\| \leq M$ for all $\alpha$;

(b) $\left\|\left(A_{\alpha}-I\right) x\right\| \rightarrow 0$ for all $x \in \bigcap_{A \in \mathbf{A}} N(A)$ and $R\left(A_{\alpha}-I\right) \subset \overline{\sum_{A \in \mathbf{A}} R(A)}$ eventually $\alpha$;

(c) For every $A \in \mathbf{A}, R\left(A_{\alpha}\right) \subset D(A)$ eventually $\alpha$ and $w$ - $\lim _{\alpha} A A_{\alpha} x=0$ for all $x \in X$, and $\left\|A_{\alpha} A x\right\| \rightarrow 0$ for all $x \in D(A)$.

Note that $\mathbf{A}$-ergodic nets with $\mathbf{A}=\{T-I ; T \in \mathbf{S}\}$ for some set $\mathbf{S} \subset B(X)$ were first studied by Eberlein [8]; such an operator net is named a right, weakly left S-ergodic net in [12, p. 75].

An abstract mean ergodic theorem from [17, Theorem 1.1] asserts that, for an A-ergodic net with $\mathbf{A}$ consisting of a single closed operator $A$, the operator $P$, defined by

$$
\left\{\begin{array}{l}
D(P):=\left\{x \in X ; s-\lim _{\alpha} A_{\alpha} x \text { exists }\right\} \\
P x=s \text { - } \lim _{\alpha} A_{\alpha} x, x \in D(P)
\end{array}\right.
$$

Received by the editors February 14, 1995 and, in revised form, May 18, 1995.

1991 Mathematics Subject Classification. Primary 47A35, 47B15, 47B44.

Key words and phrases. Abstract mean ergodic theorem, hermitian operator, hyponormal operator, $m$-dissipative operator, normal operator, orthogonality, strictly $c$-convex space.

This research was supported in part by the National Science Council of the R.O.C. 
is a bounded linear projection with norm $\|P\| \leq M$, range $R(P)=N(A)$, and null space $N(P)=\overline{R(A)}$. Applications of it to studies of ergodic properties of many particular operator families, such as integrated semigroups, cosine operator functions, and tensor product semigroups were also discussed in [18] and [19]. The purposes of this paper are: (1) to extend the above ergodic theorem to the general case that A is not a singleton; (2) to deduce from the generalized ergodic theorem Dunford's ergodic theorem [7] for multi-parameter semigroups; (3) using the abstract ergodic theorem to investigate orthogonality properties of some operators.

The generalized abstract mean ergodic theorem states as follows.

Theorem 1. Let $\mathbf{A}$ be a family of closed linear operators on $X$, and let $\left\{A_{\alpha}\right\}$ be an $\mathbf{A}$-ergodic net. Then the operator $P$ is a linear projection with norm $\|P\| \leq M$, range $R(P)=\bigcap_{A \in \mathbf{A}} N(A)$, null space $N(P)=\overline{\sum_{A \in \mathbf{A}} R(A)}$, and domain

$$
\begin{aligned}
D(P) & =\bigcap_{A \in \mathbf{A}} N(A) \oplus \overline{\sum_{A \in \mathbf{A}} R(A)} \\
& =\left\{x \in X ;\left\{A_{\alpha} x\right\} \text { has a weak cluster point }\right\} .
\end{aligned}
$$

Here $\sum_{A \in \mathbf{A}} R(A)$ denotes the linear space spanned by the spaces $R(A), A \in \mathbf{A}$. If the space $X$ is reflexive, then $D(P)=X$.

The proof of Theorem 1 and some consequences including an application to multi-parameter semigroups will be given in Section 2. Section 3 will be concerned with applications to orthogonality properties of $m$-dissipative operators and normal operators on Banach spaces.

We recall some necessary definitions. Let $X$ be a complex Banach space. The (spatial) numerical range of a linear operator $A: D(A) \subset X \rightarrow X$ is the set $W(A):=\left\{\left\langle A x, x^{*}\right\rangle ; x \in D(A), x^{*} \in X^{*},\|x\|=\left\|x^{*}\right\|=\left\langle x, x^{*}\right\rangle=1\right\}$. An operator $H \in B(X)$ is said to be hermitian if its numerical range $W(H)$ is contained in the real line $R$, or equivalently, if $\|\exp (i t H)\|=1$ for all $t \in R$ (see [2], [3]). $H$ is said to be positive if $W(H) \subset[0, \infty)$. If an operator $T$ can be expressed as $T=H+i K$ with $H$ and $K$ hermitian, then $i(H K-K H)$ is hermitian. $T$ is called normal if $T=H+i K$ for some commuting hermitian operators $H$ and $K$. These definitions generalize those familiar concepts of operators on Hilbert spaces. An operator $A: D(A) \subset X \rightarrow X$ is called dissipative if its numerical range $W(A)$ is contained in the half plane $\{z \in C, \operatorname{Re} z \leq 0\}$. A dissipative operator $A$ is called $m$-dissipative if $\rho(A) \cap(0, \infty) \neq \varnothing$.

Mattila [16] and Fong [9] proved that the null space $N(T)$ of a normal operator $T \in B(X)$ is orthogonal to the range $R(T)$ in the sense that $\|x+T y\| \geq\|x\|$ for all $x \in N(T)$ and all $y \in X$. In particular, a scalar multiple of a hermitian operator has this orthogonality property. This special case is readily contained in a theorem of Crabb and Sinclair [3, Theorem 20.6], which says that if 0 is not an interior point of the closed convex hull $\overline{c o} W(T)$ of the numerical range $W(T)$ of a bounded operator $T$ (in other words, $T$ is a scalar multiple of a bounded $m$-dissipative operator), then $\|x+T y\| \geq\|x\|-\sqrt{8\|T x\|\|y\|}$ for all $x, y \in X$.

In Section 3 we establish the following generalizations or improvements of the above known results:

(1) Let $\left\{A_{1}, A_{2}, \ldots\right\}$ be a countable family of $m$-dissipative operators or normal operators on $X$. If the resolvents of the $A_{k}$ 's are commutative, then $\bigcap_{k=1}^{\infty} N\left(A_{k}\right)$ is orthogonal to the linear span $\sum_{k=1}^{\infty} R\left(A_{k}\right)$ of $\left\{R\left(A_{k}\right)\right\}$ (Theorem 7, Corollary 8). 
(2) If $A$ is an $m$-dissipative operator, then $\|x+A y\| \geq\|x\|-2 \sqrt{\|A x\|\|y\|}$ for $x, y \in D(A)$ (Theorem 9).

(3) If 0 is not an interior point of $\overline{\mathrm{co}} W(T), T \in B(X)$, then $\|x+T y\| \geq\|x\|-$ $2 \sqrt{\|T x\|\|y\|}$ for all $x, y \in X$, and $\|T\|^{2} \leq 4\left\|T^{2}\right\|$ (Corollary 10, Corollary 11).

\section{Proof of Theorem 1 and applications to Products of $A$-ERGODIC NETS}

Proof of Theorem 1. Clearly, (a) implies that $P$ is a bounded linear operator with $\|P\| \leq M$, and both $D(P)$ and $N(P)$ are closed. The first part of (c) and the closedness of $A$ imply that $R(P) \subset N(A)$ for all $A \in \mathbf{A}$. Hence we have $R(P) \subset$ $\bigcap_{A \in \mathbf{A}} N(A)$. Conversely, if $y \in \bigcap_{A \in \mathbf{A}} N(A)$, then by the first part of (b) we have $y \in D(P)$ and $P y=y$. Therefore $R(P)=\bigcap_{A \in \mathbf{A}} N(A)$. Moreover, since $P x \in \bigcap_{A \in \mathbf{A}} N(A)$, one has $P P x=P x$ for all $x \in D(P)$, i.e. $P$ is a projection.

Next, we show that $N(P)=\overline{\sum_{A \in \mathbf{A}} R(A)}$. The second part of (c) implies that $R(A) \subset N(P)$ for all $A \in \mathbf{A}$, so that $\overline{\sum_{A \in \mathbf{A}} R(A)} \subset N(P)$. Conversely, if $x \in N(P)$, the second part of (b) implies that

$$
x=s-\lim _{\alpha}\left(I-A_{\alpha}\right) x \in \overline{\sum_{A \in \mathbf{A}} R(A)} .
$$

Therefore $N(P)=\overline{\sum_{A \in \mathbf{A}} R(A)}$.

Finally, if $x \in X$ is such that $\left\{A_{\alpha} x\right\}$ has a weakly convergent subnet $\left\{A_{\beta} x\right\}$, say $y=w-\lim _{\beta} A_{\beta} x$, it follows from the first part of (c) and the closedness of each $A \in$ A that $y \in D(A)$ and $A y=w-\lim _{\beta} A A_{\beta} x=0$. That is, $y \in \bigcap_{A \in \mathbf{A}} N(A)=R(P)$. On the other hand, the second part of (b) implies that

$$
y-x=w-\lim _{\beta}\left(A_{\beta}-I\right) x \in \overline{\sum_{A \in \mathbf{A}} R(A)}=N(P) .
$$

Hence $x=y-(y-x) \in R(P) \oplus N(P)=D(P)$, and the proof has been completed.

From Theorem 1 we now deduce some ergodic theorems for (countable) products of $A$-ergodic nets.

Corollary 2. Let $\left\{A^{(k)}\right\}$ be a sequence of closed linear operators on X. For each $k$, let $\left\{A_{\alpha}^{(k)}\right\}$ be an $A^{(k)}$-ergodic net satisfying $\infty$;

$\left(\mathrm{a}^{\prime}\right) M:=\sup \left\{\left\|\prod_{k \in F} A_{\alpha}^{(k)}\right\| ; F\right.$ a finite set of natural numbers and for all $\left.\alpha\right\}<$

$\left(\mathrm{b}^{\prime}\right)\left\|\left(A_{\alpha}^{(k)}-I\right) x\right\| \rightarrow$ for all $x \in N\left(A^{(k)}\right)$ and $R\left(A_{\alpha}^{(k)}-I\right) \subset \overline{R\left(A^{(k)}\right)}$ for all $\alpha$;

(c') $R\left(A_{\alpha}^{(k)}\right) \subset D\left(A^{(k)}\right)$ and $A_{\alpha}^{(k)} A^{(k)} \subset A^{(k)} A_{\alpha}^{(k)}$ for all $\alpha$, and $\left\|A^{(k)} A_{\alpha}^{(k)}\right\| \rightarrow 0$.

$\left(\mathrm{d}^{\prime}\right) A_{\alpha}^{(j)} A_{\alpha}^{(k)}=A_{\alpha}^{(k)} A_{\alpha}^{(j)}$ for all $\alpha, j$, and $k$.

For a nondecreasing net $\left\{r_{\alpha}\right\}$ of positive integers, let $P: D(P) \subset X \rightarrow X$ be the operator defined by

$$
\left\{\begin{array}{l}
D(P):=\left\{x \in X ; s-\lim _{\alpha} A_{\alpha}^{(1)} A_{\alpha}^{(2)} \cdots A_{\alpha}^{\left(r_{\alpha}\right)} x \text { exists }\right\} \\
P x=s-\lim _{\alpha} A_{\alpha}^{(1)} A_{\alpha}^{(2)} \cdots A_{\alpha}^{\left(r_{\alpha}\right)} x, x \in D(P)
\end{array}\right.
$$

Then $P$ is a linear projection with norm $\|P\| \leq M$, range $R(P)=\bigcap_{k=1}^{\infty} N\left(A^{(k)}\right)$, null space $N(P)=\overline{\sum_{k=1}^{\infty} A^{(k)}}$, and domain

$$
D(P)=\left\{x \in X ;\left\{A_{\alpha}^{(1)} A_{\alpha}^{(2)} \cdots A_{\alpha}^{\left(r_{\alpha}\right)} x\right\} \text { has a weak cluster point }\right\} .
$$


Proof. Define $A_{\alpha}:=A_{\alpha}^{(1)} A_{\alpha}^{(2)} \cdots A_{\alpha}^{\left(r_{\alpha}\right)}$ for all $\alpha$. It suffices to check that the net $\left\{A_{\alpha}\right\}$ is an $\mathbf{A}$-ergodic net with $\mathbf{A}=\left\{A^{(k)}\right\}$, i.e. (a)-(c) of Theorem 1 are satisfied. $\left(\mathrm{a}^{\prime}\right)$ implies (a). For every $x \in X$ and $\alpha$, we have, by the commutativity of $A_{\alpha}^{(j)}$ and $A_{\alpha}^{(k)}$, that

$$
\begin{aligned}
\left(A_{\alpha}-I\right) x & =A_{\alpha}^{(1)} A_{\alpha}^{(2)} \cdots A_{\alpha}^{\left(r_{\alpha}\right)} x-x \\
& =\sum_{k=2}^{r_{\alpha}} A_{\alpha}^{(1)} A_{\alpha}^{(2)} \cdots A_{\alpha}^{(k-1)}\left(A_{\alpha}^{(k)}-I\right) x+\left(A_{\alpha}^{(1)}-I\right) x \\
& =\sum_{k=2}^{r_{\alpha}}\left(A_{\alpha}^{(k)}-I\right) A_{\alpha}^{(1)} A_{\alpha}^{(2)} \cdots A_{\alpha}^{(k-1)} x+\left(A_{\alpha}^{(1)}-I\right) x,
\end{aligned}
$$

from which and $\left(\mathrm{a}^{\prime}\right)$ it is seen that parts 1 and 2 of (b) follow respectively from the corresponding parts of $\left(b^{\prime}\right)$.

Also we have for every $k$

$$
A_{\alpha}=A_{\alpha}^{(1)} A_{\alpha}^{(2)} \cdots A A_{\alpha}^{\left(r_{\alpha}\right)}=A_{\alpha}^{(k)} \prod_{1 \leq j \neq k \leq r_{\alpha}} A_{\alpha}^{(j)}=\left\{\prod_{1 \leq j \neq k \leq r_{\alpha}} A_{\alpha}^{(j)}\right\} A_{\alpha}^{(k)},
$$

which and ( $\left.\mathrm{c}^{\prime}\right)$ imply that $R\left(A_{\alpha}\right) \subset D\left(A^{(k)}\right)$,

$$
\begin{aligned}
& \left\|A^{(k)} A_{\alpha}\right\| \leq\left\|A^{(k)} A_{\alpha}^{(k)}\right\| \prod_{1 \leq j \neq k \leq r_{\alpha}} A_{\alpha}^{(j)} \| \rightarrow 0, \\
& \left\|A_{\alpha} A^{(k)} x\right\| \leq\left\|\prod_{1 \leq j \neq k \leq r_{\alpha}} A_{\alpha}^{(j)}\right\|\left\|A_{\alpha}^{(k)} A^{(k)}\right\|\|x\| \rightarrow 0, \quad x \in D\left(A^{(k)}\right) .
\end{aligned}
$$

Therefore $\left\{A_{\alpha}\right\}$ is an $\mathbf{A}$-ergodic net, and the conclusion now follows from Theorem 1.

In the special cases $r_{\alpha} \equiv m$ and $r_{n}=n$, Corollary 2 becomes the following two corollaries.

Corollary 3. Let $\left\{A^{(k)}\right\}, k=1,2, \ldots, m$, be closed linear operators on $X$. For each $k$, let $\left\{A_{\alpha}^{(k)}\right\}$ be an $A^{(k)}$-ergodic net satisfying conditions $\left(\mathrm{b}^{\prime}\right),\left(\mathrm{c}^{\prime}\right)$, and $\left(\mathrm{d}^{\prime}\right)$. Let $P: D(P) \subset X \rightarrow X$ be the operator defined by

$$
\left\{\begin{array}{l}
D(P):=\left\{x \in X ; s-\lim _{\alpha} A_{\alpha}^{(1)} A_{\alpha}^{(2)} \cdots A_{\alpha}^{(m)} x \text { exists }\right\} \\
P x=s-\lim _{\alpha} A_{\alpha}^{(1)} A_{\alpha}^{(2)} \cdots A_{\alpha}^{(m)} x, x \in D(P) .
\end{array}\right.
$$

Suppose that $\left\|A_{\alpha}^{(k)}\right\| \leq M_{k}$ for all $1 \leq k \leq m$. Then $P$ is a linear projection with norm $\|P\| \leq M_{1} \cdots M_{m}$, range $R(P)=\bigcap_{k=1}^{m} N\left(A^{(k)}\right)$, null space $N(P)=$ $\overline{\sum_{k=1}^{m} A^{(k)}}$, and domain

$$
D(P)=\left\{x \in X ;\left\{A_{\alpha}^{(1)} A_{\alpha}^{(2)} \cdots A_{\alpha}^{(m)} x\right\} \text { has a weak cluster point }\right\} .
$$

Corollary 4. Let $\left\{A^{(k)}\right\}, k=1,2, \ldots$, be a sequence of closed linear operators on $X$. For each $k$, let $\left\{A_{n}^{(k)}\right\}$ be an $A^{(k)}$-ergodic sequence satisfying $\left(\mathrm{b}^{\prime}\right),\left(\mathrm{c}^{\prime}\right),\left(\mathrm{d}^{\prime}\right)$ (with $\alpha$ replaced by $n$ ). Let $P: D(P) \subset X \rightarrow X$ be the operator defined by

$$
\left\{\begin{array}{l}
D(P):=\left\{x \in X ; s-\lim _{n} A_{n}^{(1)} A_{n}^{(2)} \cdots A_{n}^{(n)} x \text { exists }\right\} \\
P x=s-\lim _{n} A_{n}^{(1)} A_{n}^{(2)} \cdots A_{n}^{(n)} x, x \in D(P) .
\end{array}\right.
$$


Suppose that $M:=\sup \left\{\left\|\prod_{k \in F} A_{n}^{(k)}\right\| ; F\right.$ a finite set of natural numbers and $n \geq$ $1\}<\infty$. Then $P$ is a linear projection with norm $\|P\| \leq M$, range $R(P)=$ $\bigcap_{k=1}^{\infty} N\left(A^{(k)}\right)$, null space $N(P)=\overline{\sum_{k=1}^{\infty} A^{(k)}}$, and domain

$$
D(P)=\left\{x \in X ;\left\{A_{n}^{(1)} A_{n}^{(2)} \cdots A_{n}^{(n)} x\right\} \text { has a weak cluster point }\right\} .
$$

Next, we give two illustrative examples.

Example 1. Let $A^{(1)}, A^{(2)}, \ldots$ be a sequence of closed operators on a Banach space $X$ satisfying $(0, \infty) \subset \rho\left(A^{(k)}\right),\left(\lambda-A^{(j)}\right)^{-1}\left(\lambda-A^{(k)}\right)^{-1}=\left(\lambda-A^{(k)}\right)^{-1}\left(\lambda-A^{(j)}\right)^{-1}$, and $\left\|\lambda\left(\lambda-A^{(k)}\right)^{-1}\right\| \leq M_{k}$ for all $\lambda>0$ and $j, k=1,2, \ldots$. Suppose further that $M=\sup \left\{\prod_{k \in F} M_{k} ; F\right.$ a finite set of natural numbers $\}<\infty$ (particularly, $M_{k}=1$ for all $\left.k\right)$. If we put $A_{n}^{(k)}:=\frac{1}{n}\left(\frac{1}{n}-A^{(k)}\right)^{-1}$ for $n \geq 1$, then we have $A_{n}^{(k)} A^{(k)} \subset A^{(k)} A_{n}^{(k)}=\frac{1}{n}\left(A_{n}^{(k)}-I\right)$, so that conditions in Corollary 4 are satisfied. Hence we can formulate the next theorem.

Theorem 5. Under the above assumption on closed operators $A^{(1)}, A^{(2)}, \ldots$, the limits $\lim _{n \rightarrow \infty} n^{-n}\left(\frac{1}{n}-A^{(1)}\right)^{-1} \cdots\left(\frac{1}{n}-A^{(n)}\right)^{-1} x$ define a linear projection $P$ with norm $\|P\| \leq M$, range $R(P)=\bigcap_{k=1}^{\infty} N\left(A^{(k)}\right)$, null space $N(P)=\overline{\sum_{k=1}^{\infty} R\left(A^{(k)}\right)}$, and domain

$$
\begin{aligned}
D(P)= & {\left[\bigcap_{k=1}^{\infty} N\left(A^{(k)}\right)\right] \oplus \overline{\sum_{k=1}^{\infty} R\left(A^{(k)}\right)} } \\
= & \left\{x \in X ; \exists n_{k} \rightarrow \infty \ni w-\lim _{k \rightarrow \infty} n_{k}^{-n_{k}}\left(\frac{1}{n_{k}}-A^{(1)}\right)^{-1}\right. \\
& \left.\cdots\left(\frac{1}{n_{k}}-A^{\left(n_{k}\right)}\right)^{-1} x \text { exists }\right\} .
\end{aligned}
$$

Example 2. For $\alpha, \beta$ in Euclidean $N$-space $R^{N}, \alpha>\beta$ means that $\alpha_{1}>\beta_{1}, \ldots, \alpha_{N}$ $>\beta_{N}$; and $\alpha \rightarrow \infty$ means that $\alpha_{1} \rightarrow \infty, \ldots, \alpha_{N} \rightarrow \infty$. Let $\left\{T(t) ; t \in R^{N}, t>0\right\} \subset$ $B(X)$ be a strongly continuous semigroup (see [11]) such that $\|T(u)\| \leq M$ for all $0<u \in R^{N}$. The averages $A(\alpha) ; \alpha=(a, \ldots, a)$, are defined in terms of $T(u)$ and the $N$-dimensional interval $\sigma(\alpha) \equiv(0, a]^{N}$, by the equation

$$
A(\alpha) x=\frac{1}{a^{N}} \int_{\sigma(\alpha)} T(u) x d u, \quad x \in X .
$$

Let $\mathbf{A}=\left\{T(t)-I ; t \in R^{N}, t>0\right\}$.

Since $(A(\alpha)-I) x=\frac{1}{a^{N}} \int_{\sigma(\alpha)}(T(u)-I) x d u,\{A(\alpha)\}$ satisfies condition (b). Since

$$
(T(t)-I) A(\alpha) x=\frac{1}{a^{N}}\left[\int_{\sigma(\alpha)^{\prime} \cap(t+\sigma(\alpha))} T(u) x d u-\int_{\sigma(\alpha) \cap(t+\sigma(\alpha))^{\prime}} T(u) x d u\right],
$$

and since the measures of the sets $\sigma(\alpha)^{\prime} \cap(t+\sigma(\alpha))$ and $\sigma(\alpha) \cap(t+\sigma(\alpha))^{\prime}$ are both $O\left(a^{N-1}\right)$ as $\alpha \rightarrow \infty$ (see [7]), we have that $\|(T(t)-I) A(\alpha) x\| \rightarrow 0$ as $\alpha \rightarrow \infty$. Hence $\{A(\alpha)\}$ is an $\mathbf{A}$-ergodic net.

In case the semigroup $T(\cdot)$ is strongly continuous on $\left\{\alpha \in R^{N} ; \alpha_{k} \geq 0,0 \leq k \leq\right.$ $N\}$ (i.e. an $N$-parameter $C_{0}$-semigroup), the families $T_{k}(\cdot):=\left\{T\left(t e_{k}\right) ; 0 \leq t<\right.$ $\infty\}, k=1, \ldots, N$, are commuting one-parameter $C_{0}$-semigroups. Let $A_{k}$ be the infinitesimal generator of $T_{k}(\cdot)$. It can be verified that $\{A(\alpha)\}$ is an $\mathbf{A}$-ergodic net with $\mathbf{A}=\left\{A_{1}, A_{2}, \ldots, A_{N}\right\}$. 
Now we can deduce the following theorem from Theorem 1.

Theorem 6. Let $\left\{T(t) ; t \in R^{N}, t>0\right\}$ be a uniformly bounded, strongly continuous semigroup. The operator $P: x \rightarrow \lim _{\alpha \rightarrow \infty} A(\alpha) x$ is a bounded linear projection with range $R(P)=\bigcap_{0<u \in R^{N}} N(T(u)-I)$, null space $N(P)=\overline{\bigcup_{0<u \in R^{N}} R(T(u)-I)}$, and domain

$$
D(P)=\{x \in X ;\{A(\alpha) x\} \text { has a weak cluster point }\} .
$$

In case that $T(\cdot)$ is a uniformly bounded $N$-parameter $C_{0}$-semigroup, one also has $R(P)=\bigcap_{k=1}^{N} N\left(A_{k}\right)$ and $N(P)=\overline{\sum_{k=1}^{N} R\left(A_{k}\right)}$.

\section{ORThOGONALITY PROPERTIES OF SOME OPERATORS}

Let $Y$ and $Z$ be two subspaces of the Banach space $X$. We say that $Y$ is orthogonal to $Z$, and denote this by $Y \perp Z$, if $\|y+z\| \geq\|y\|$ for all $y \in Y$ and $z \in Z$. This definition of orthogonality is consistent with the usual concept of orthogonality in Hilbert spaces, and is equivalent to $Y \cap Z=\{0\}$ and the projection $P$ onto $Y$ along $Z$ has norm $\|P\|=1$.

Theorem 7. Let $\left\{A_{n}\right\}$ be a sequence of $m$-dissipative operators such that their resolvents are commutative. Then

$$
\left[\bigcap_{k=1}^{\infty} N\left(A_{k}\right)\right] \perp \overline{\sum_{k=1}^{\infty} R\left(A_{k}\right) .}
$$

If the space $X$ is reflexive, then

$$
X=\left[\bigcap_{k=1}^{\infty} N\left(A_{k}\right)\right] \stackrel{\perp}{\oplus \sum_{k=1}^{\infty} R\left(A_{k}\right)} .
$$

Proof. Since an operator $A$ is dissipative if and only if for each $\lambda>0, \lambda-A$ is injective and $\left\|\lambda(\lambda-A)^{-1}\right\| \leq 1$ (see [10, p. 26]), the theorem follows immediately from Theorem 5. Noting that $A$ and $\lambda(\lambda-A)^{-1}-I(\lambda>0)$ have the same null space and range, one can also deduce the theorem directly from Corollary 4 by setting $A^{(k)}=\lambda\left(\lambda-A_{k}\right)^{-1}-I$ and $A_{n}^{(k)}=\frac{1}{n} \sum_{j=0}^{n-1}\left(\lambda\left(\lambda-A_{k}\right)^{-1}\right)^{j}$.

The conclusion of Theorem 7 holds in particular for any sequence of commutative hermitian operators, because $i$ times a hermitian operator is $m$-dissipative. The next corollary shows that normal operators share the same property. For the proof of it we need the generalized Fuglede theorem (see [5], [6], [9]), which states that if $T=H+i K$ is a normal operator, where $H$ and $K$ are commuting hermitian operators, then $N(T)=N(H) \cap N(K)$. Applying this to the normal derivation $\Delta_{T, T}=\Delta_{H, H}+i \Delta_{K, K}$ (see [1], [13], [17]), one obtains the Fuglede theorem for normal operators on Banach spaces, that is, $T B=B T$ if and only if $H B=B H$ and $K B=B K$.

Corollary 8. Let $\left\{T_{n}\right\}$ be a sequence of commuting normal operators on X. Then

$$
\left[\bigcap_{k=1}^{\infty} N\left(T_{k}\right)\right] \perp \overline{\sum_{k=1}^{\infty} R\left(T_{k}\right)} .
$$


If the space $X$ is reflexive, then

$$
X=\left[\bigcap_{k=1}^{\infty} N\left(T_{k}\right)\right] \oplus \bar{\oplus} \overline{\sum_{k=1}^{\infty} R\left(T_{k}\right)} .
$$

Proof. Since normal operators $T_{k}=H_{k}+i K_{k}, k=1,2, \ldots$, are commutative, the above observation shows that the hermitian operators $H_{1}, H_{2}, \ldots, K_{1}, K_{2}, \ldots$, are commutative. It follows from Theorem 7 that

$$
\left\{\bigcap_{k=1}^{\infty}\left[N\left(H_{k}\right) \cap N\left(K_{k}\right)\right]\right\} \perp \overline{\sum_{k=1}^{\infty}\left[R\left(H_{K}\right)+R\left(K_{k}\right)\right]},
$$

and in case $X$ is reflexive,

$$
X=\left\{\bigcap_{k=1}^{\infty}\left[N\left(H_{k}\right) \cap N\left(K_{k}\right)\right]\right\} \oplus \bar{\oplus} \overline{\sum_{k=1}^{\infty}\left[R\left(H_{k}\right)+R\left(K_{k}\right)\right]} .
$$

Since $N\left(T_{k}\right)=N\left(H_{k}\right) \cap N\left(K_{k}\right)$ and $R\left(T_{k}\right) \subset R\left(H_{k}\right)+R\left(K_{k}\right)$, we have proved

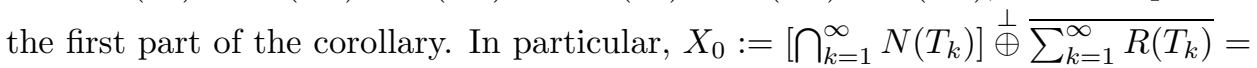
${ }^{\perp}\left[\sum_{k=1}^{\infty} R\left(T_{k}^{*}\right)\right] \stackrel{\perp}{\oplus} \overline{\sum_{k=1}^{\infty} R\left(T_{k}\right)}$ is a closed linear subspace of $X$.

Since the dual operators $T_{k}^{*}$ are also normal operators, the first part of this theorem implies that

$$
\left[\bigcap_{k=1}^{\infty} N\left(T_{k}^{*}\right)\right] \perp \overline{\sum_{k=1}^{\infty} R\left(T_{k}^{*}\right)}
$$

If $X$ is reflexive, then $X_{0}^{\perp}=\overline{\sum_{k=1}^{\infty} R\left(T_{k}^{*}\right)} \cap\left[\bigcap_{k=1}^{\infty} N\left(T_{k}^{*}\right)\right]=\{0\}$. Hence $X_{0}=X$, i.e. (3.3) holds.

Remarks. (1) In the case of bounded operators, Theorem 7 and Corollary 8 both follow also from [14, Theroem 3.3] and [15, Theorem 2].

(2) If $X$ is smooth, i.e. the norm of $X$ is Gâteaux differentiable, then, because $x \perp y$ and $x \perp z$ imply $x \perp y+z$, the first part of Theorem 7 and that of Corollary 8 follow immediately from the case of a single operator, even when the resolvents of the concerned family of operators are not commutative.

(3) Mattila [15] showed that if $T=H+i K$ is a hyponormal operator on a strictly $c$-convex Banach space, then $N(T)=N(H) \cap N(K)$. Thus when $X$ is a smooth and strictly $c$-convex Banach space (e.g. $L_{p}, 1<p<\infty$, or Hilbert spaces), the first part of Corollary 8 also holds for noncommutative hypernormal operators.

Theorem 7 implies that if $A$ is an $m$-dissipative operator, then $\|x+A y\| \geq\|x\|$ for all $x \in N(A)$ and $y \in D(A)$. The next theorem gives a generalized inequality.

Theorem 9. If $A$ is an $m$-dissipative operator in a Banach space $X$, then

$$
\|x+A y\| \geq\|x\|-2 \sqrt{\|A x\|\|y\|} \text { for all } x, y \in D(A) .
$$

Proof. For each $n \geq 1$ we define an operator function $T_{n}(\cdot)$ by

$$
T_{n}(t) x:=t^{-1} \int_{0}^{t}\left(I-\frac{s}{n} A\right)^{-n} x d s, \quad x \in X, t>0 .
$$


The $m$-dissipativity of $A$ implies $\left\|T_{n}(t)\right\| \leq 1$ for all $n$ and $t$. Then we have for $x, y \in D(A), n \geq 2$ and $t>0$

$$
\begin{aligned}
\|x+A y\| \geq & \left\|T_{n}(t)(x+A y)\right\| \\
& \geq\|x\|-\left\|\left(T_{n}(t)-I\right) x+T_{n}(t) A y\right\| \\
& \geq\|x\|-\left\|\frac{1}{t} \int_{0}^{t}\left[\left(I-\frac{s}{n} A\right)^{-n}-I\right] x d s\right\|-\left\|\frac{1}{t} \int_{0}^{t}\left(I-\frac{s}{n} A\right)^{-n} A y d s\right\| \\
= & \|x\|-\left\|\frac{1}{t} \int_{0}^{t} \frac{s}{n} \sum_{k=0}^{n-1}\left(I-\frac{s}{n} A\right)^{-k-1} A x d s\right\| \\
& -\left\|\frac{1}{t} \int_{0}^{t} \frac{n}{n-1} \frac{d}{d s}\left[\left(I-\frac{s}{n} A\right)^{1-n} y\right] d s\right\| \\
\geq & \|x\|-\frac{1}{t} \int_{0}^{t} s d s\|A x\|-\left\|\frac{1}{t} \frac{n}{n-1}\left[\left(I-\frac{t}{n} A\right)^{1-n} y-y\right]\right\| \\
\geq & \|x\|-\left(\frac{t}{2}\|A x\|+\frac{n}{n-1} \frac{2}{t}\|y\|\right) .
\end{aligned}
$$

Hence $\|x+A y\| \geq\|x\|-\left(\frac{t}{2}\|A x\|+\frac{2}{t}\|y\|\right)$ for all $t>0$. Minimizing the function $\frac{t}{2}\|A x\|+\frac{2}{t}\|y\|$, we obtain its minimum $2 \sqrt{\|A x\|\|y\|}$, and hence the estimate (3.4).

Theorem 9 contains as a corollary the following slight improvement of a theorem of Crabb and Sinclair (see [4] or [3, Theorem 20.6]).

Corollary 10. If an operator $T \in B(X)$ is such that 0 is not an interior point of $\overline{\mathrm{co}} W(T)$, then $\|x+T y\| \geq\|x\|-2 \sqrt{\|T x\|\|y\|}$ for all $x, y \in X$.

Using Corollary 10 one can deduce some consequences. For instance, by modifying the proof of Corollary 20.12 in [3] we obtain the following improvement.

Corollary 11. Under the hypothesis of Corollary 10, the inequality $\|T\|^{2} \leq 4\left\|T^{2}\right\|$ holds.

\section{ACKNOWLEDGMENT}

The authors would like to thank the referee for his valuable suggestions.

\section{REFERENCES}

1. J. Anderson, On normal derivations, Proc.Amer. Math. Soc. 38 (1973), 135-140. MR 47:875

2. F. F. Bonsall and J. Duncan, Numerical Ranges of Operators on Normed Spaces and of Elements of Normed Algebras, London Math. Soc. Lecture Note Series No. 2, Cambridge Univ. Press, Cambridge, 1971. MR 44:5779

3. _ Numerical Ranges II, London Math. Soc. Lecture Note Series No. 10, Cambridge Univ. Press, Cambridge, 1973. MR 56:1063

4. M. J. Crabb and A. M. Sinclair, On the boundary of the spatial numerical range, Bull. London Math. Soc. 4 (1972), 17-19. MR 46:7929

5. M. J. Crabb and P. G. Spain, Commutators and normal operators, Glasgow Math. J. 18 (1977), 197-198. MR 56:1115

6. H. R. Dawson, T. A. Gillespie, and P. G. Spain, A commutativity theorem for hermitian operators, Math. Ann. 220 (1976), 215-217. MR 54:5898

7. N. Dunford, Some ergodic theorems, Proc. Royal Soc. Edinburgh 85A (1980), 111-118. MR 82a: 47006 
8. W. Eberlein, Abstract ergodic theorems and weak almost periodic functions, Trans. Amer. Math. Soc. 67 (1949), 217-240. MR 12:112a

9. C. K. Fong, Normal operators on Banach spaces, Glasgow Math. J. 20 (1979), 163-168. MR 80e: 47020

10. J. A. Goldstein, Semigroups of Linear Operators and Applications, Oxford University Press, Oxford, 1985. MR 87c:47056

11. E. Hille and R. S. Phillips, Functional Analysis and Semi-groups, Amer. Math. Soc. Colloq. Publ., Vol. 31, Amer. Math. Soc., Providence, R.I., 1957. MR 19:664d

12. U. Krengel, Ergodic Theorems, Walter de Gruyter, Berlin, 1985. MR 87i:28001

13. J. Kyle, Numerical ranges of derivations, Proc. Edinburgh Math. Soc. 21 (1978), 33-39. MR 58:7127

14. K. Mattila, Normal operators and proper boundary points of the spectra of operators on a Banach space, Ann. Acad. Sci. Fenn. Ser. A I Math. Dissertationes 19 (1978), 48 pp. MR 80j: 47031

15. - On proper boundary points of the spectrum and complemented eigenspaces, Math. Scand. 43 (1978), 363-368. MR 80i:47003

16. Complex strict and uniform convexity and hyponormal operators, Math. Proc. Camb. Phil. Soc. 96 (1984), 483-493. MR 86c:47026

17. S.-Y. Shaw, On numerical ranges of generalized derivations and related properties, J. Austral. Math. Soc. (series A) 36 (1984), 134-142. MR 85e:47001

18. 428-441. MR 90k:47018

19. Uniform convergence of ergodic limits and approximate solutions, Proc. Amer. Math. Soc. 114 (1992), 405-411. MR 92e:47012

Department of Mathematics, National Central University, Chung-Li, Taiwan 320 Current address, Y.-C. Li: Department of Mathematics, Chung Yuan University, Chung-Li, Taiwan 320

E-mail address: shaw@math.ncu.edu.tw 